\title{
MAGNETIC CAPPING LAYER EFFECT OF PtCo ALLOY FILM COUPLED WITH TbFeCo
}

\author{
S. OHNUKI, K. SHIMAZAKI, N. OHTA and H. FUJIWARA \\ Tsukuba Research Laboratory, Hitachi Maxell, Ltd., Mitsukaido, Ibatraki 300-25, Japan
}

\begin{abstract}
A new Magneto-Optical disk using a TbFeCo film capped with in-plane magnetizing PtCo alloy film was investigated. We found a magnetic capping layer effect resulting in small switching field which means a small external field required for complete writing and erasing. This technology is very useful for overwriting schemes such as in field modulation type and two beam type. The results from the written domain observation suggest that the above phenomenon originates from the suppression of the nucleation of microdomains in the interior of the laser-written mark during the switching process due to the exchange coupled PtCo layer.

KEYWORDS:MAGNETIC CAPPING LAYER EFFECT, PtCo ALLOY, SWITCHING FIELD, MICRODOMAINS, EXCHANGE COUPLING, IN-PLANE MAGNETIZING LAYER
\end{abstract}

\section{INTRODUCTION}

For the next generation Magneto-Optical(MO) disk, overwritable media are required to achieve higher data transfer rate. A lot of overwriting schemes have been proposed, among them, field modulation type and two beam type require the key technology to make the switching field as small as possible. Here, we report a new MO disk using a TbFeCo film capped with an in-plane magnetizing layer. In such a new disk, we obtained the small switching field. As a magnetic capping layer, PtCo alloy system was studied[1,2]. The control of saturation magnetization or curie temperature is easy in this alloy. It has both a high reflectivity to increase optical efficiency and a high corrosion resistance.

\section{EXPERIMENTAL}

$\mathrm{PtCo} / \mathrm{TbFeCo}$ double layered film, sandwiched by nitride protective layers, was deposited on 5.25 inch diameter glass-2P substrates by $\mathrm{rf}$ magnetron sputtering using a $\mathrm{TbFeCo}$ composite target and a $\mathrm{Pt}$ target with Co pellets. The back pressure, sputtering gas(Ar) pressure and $\mathrm{rf}$ input power were $4 \times$ $10^{-6}$ Torr, $5 \times 10^{-3}$ Torr and $4.39 \mathrm{~W} / \mathrm{cm}^{2}$ respectively. A sample with a layer structure such as (Al alloy/nitride/ $\mathrm{TbFeCo} /$ nitride/substrate) was also used as a conventional standard to compare with that of $\mathrm{PtCo} / \mathrm{TbFeCo}$ film. The composition of $\mathrm{PtCo}$ alloy and $\mathrm{TbFeCo}$ were determined by a X-ray micro analysis (XMA) and an inductively coupled plasma spectroscopy(ICPS) respectively. The magnetic properties of films were measured with a vibrating sample magnetometer and a torque magnetometer in the applied fields up to $16 \mathrm{kOe}$. Curie temperature of $\mathrm{TbFeCo}$ film used in this study was $220^{\circ} \mathrm{C}$ and compensation temperature $140-180^{\circ} \mathrm{C}$. The dynamic recording performance in those $\mathrm{MO}$ disks were investigated using a conventional MO drive with an objective lens of 0.52 numerical aperture and 830 $\mathrm{nm}$ wavelength laser. The measurements of the external field dependence of the carrier and noise levels were carried out under the conditions of $7.54 \mathrm{~m} / \mathrm{sec}$ linear velocity, .60nsec pulse width, $4.93 \mathrm{MHz}$ recording frequency, $5-7 \mathrm{~mW}$ recording laser power and $1.5 \mathrm{~mW}$ reading power. The corresponding written domains were observed by using a polarized optical microscope with 100 times oil-immersion objective lens.

\section{RESULTS AND DISCUSSION}

The sputtered PtCo alloy films exhibited ferromagnetism in the region of $0-92 a t \% \mathrm{Pt}$ content. Figure 1 shows saturation magnetization (Ms) and coercivity (Hc) of PtCo alloy films with a thickness of 100-150 nm as a function of $\mathrm{Pt}$ content. These values were obtained from the easy axis M-H

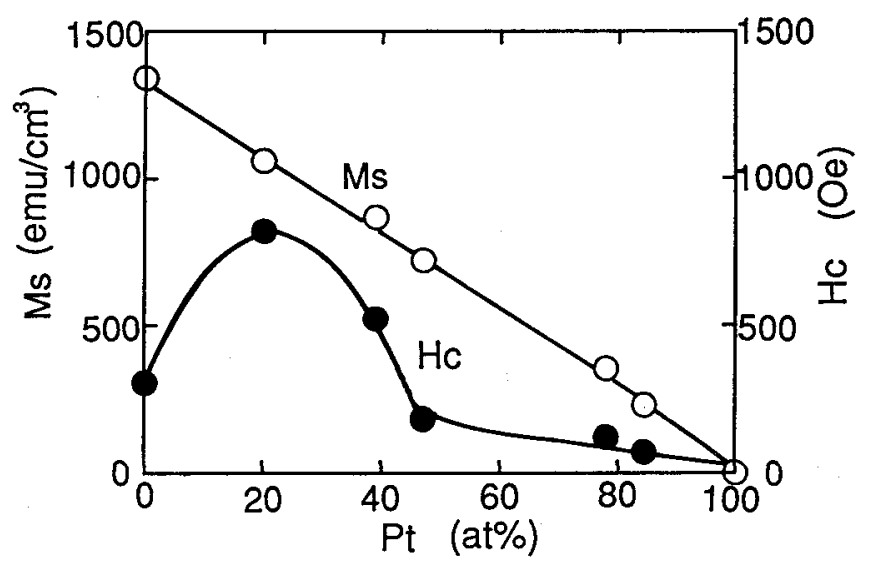

Fig. 1 Saturation magnetization Ms and coercivity $\mathrm{Hc}$ of PtCo alloy films as a function of Pt content. 
loops at the room temperature. The saturation magnetization decreases linearly with increasing $\mathrm{Pt}$ content, and coercivity has a maximum at about 20 at\% Pt. The dependence of coercivity on the composition is in qualitative agreement with the data obtained by M.Kitada, et al[2]. The easy axis of $\mathrm{PtCo}$ alloy film is parallel to the film plane. As shown in Fig. 2, magnetic anisotropy energy ( $\left.\mathrm{K}_{/ 1}\right)$ decreases monotonously with the increase of $\mathrm{Pt}$ content, and the values are almost the same as the shape anisotropy energy $\left(2 \pi \mathrm{Ms}^{2}\right)$. Curie temperature Tc which was obtained through a temperature dependence of Ms decreases with the addition of $\mathrm{Pt}$ as shown in Fig.3.

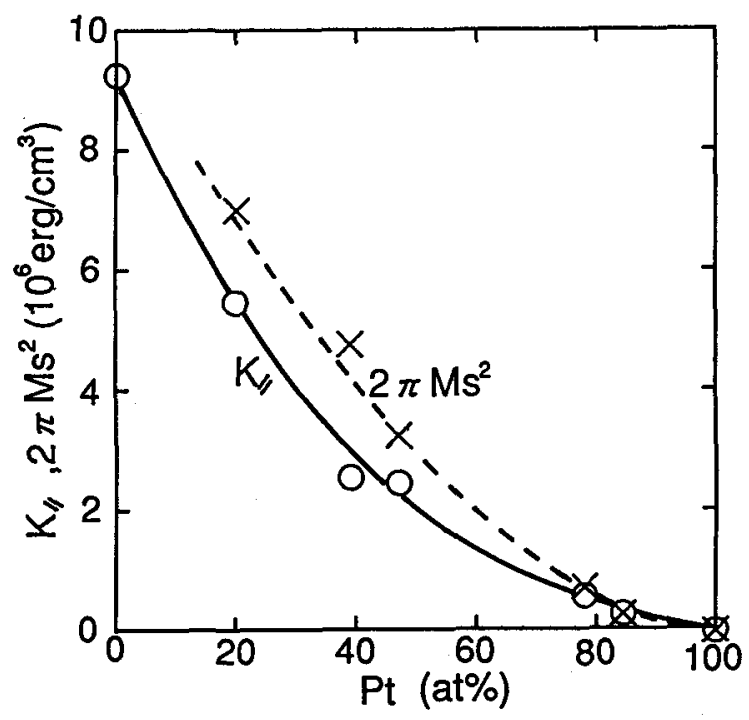

Fig.2 Magnetic anisotropy energy $K$, and shape anisotropy energy $2 \pi \mathrm{Ms}^{2}$ of PtCo alloy films as a function of Pt content.

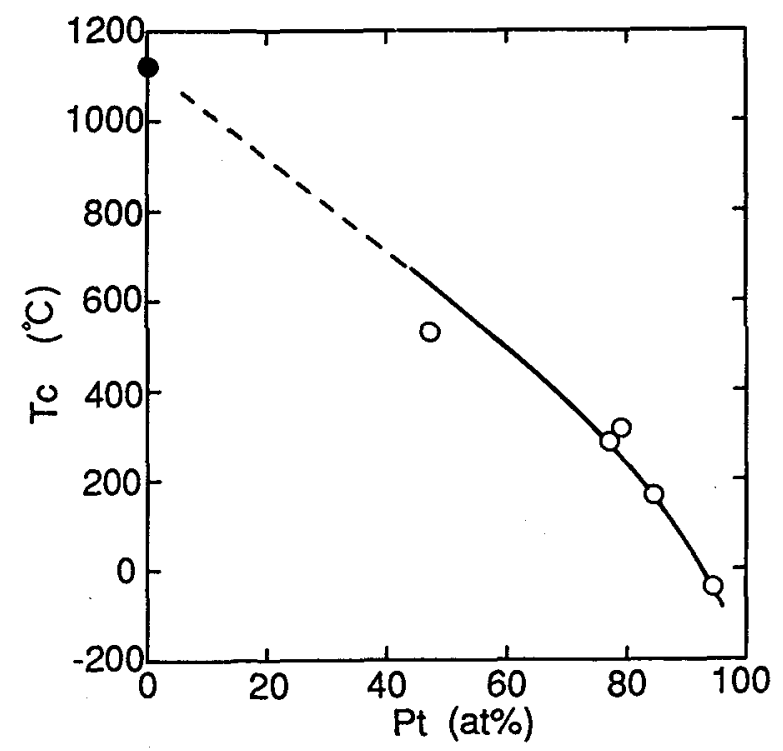

Fig.3 Curie temperature Tc of PtCo alloy films as a function of content. The solid circle indicates Tc of pure Co bulk described in ref.3.
The external field dependence of the carrier and noise levels for the $\mathrm{MO}$ disks using $\mathrm{TbFeCo}$ film capped with PtCo alloy films, which has the abovementioned magnetic properties, is shown in Fig.4. Magnetic layers of the disk $A$ and the disk $B$ are $\mathrm{Pt}_{84} \mathrm{Co}_{16} / \mathrm{TbFeCo}$ and $\mathrm{Pt}_{20} \mathrm{Coso} / \mathrm{TbFeCo}$, respectively. The disk $\mathrm{C}$ is a conventional structure one with a single TbFeCo layer. The carrier level of the disk $C$ increases gradually with increasing writing field (positive sign), and decreases gradually with the increase of erasing field (negative). In this case, the external field over $600 \mathrm{Oe}$ is required for complete writing and erasing. On the other hand, in the disk $A$, abrupt changes in the carrier and noise levels occur near zero field. A field strength of $100 \mathrm{Oe}$ is sufficient for complete writing, and minus $150 \mathrm{Oe}$ for erasing. Those results depend on the composition of the PtCo alloy film. The disk B does not show any abrupt change near zero field.

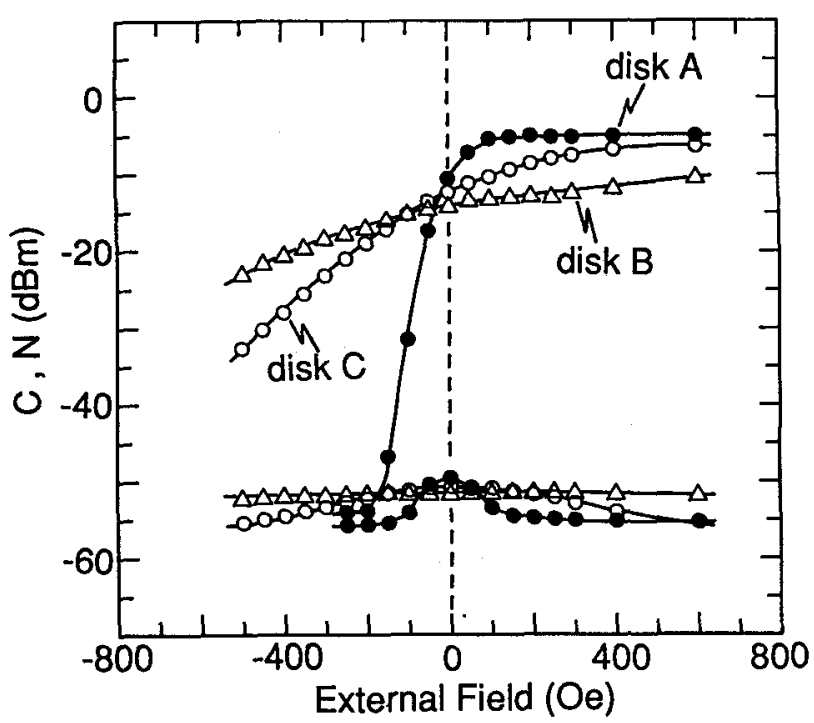

Fig.4. External field dependence of the carrier and noise levels for $\operatorname{disk} A$,diskB and diskC.

The detailed PtCo composition dependence is shown in Fig.5. Here, $\left|\mathrm{C}_{-200-\mathrm{C}_{600}}\right|$ means a carrier level difference between plus $600 \mathrm{Oe}$ and minus $200 \mathrm{Oe}$. It was introduced as a rough measure of the erasability using a PtCo capping layer. Figure 5 shows a remarkable erasability near the composition of 80 $90 \mathrm{at} \% \mathrm{Pt}$. The dependence of $\left|\mathrm{C}_{-200}-\mathrm{C}_{600}\right|$ on a layer thickness of $\mathrm{Pt}_{84} \mathrm{Co}_{16}$ was also investigated in the thickness range of $2-70 \mathrm{~nm}$. The PtCo capping effect was observed even at only $2 \mathrm{~nm}$ thickness, and was almost the same in the PtCo layer thickness range studied.

In order to investigate such a capping layer effect more in detail, we observed written domains. Photograph 1 shows the external field dependence of written domains for the disk $A$ and the disk $C$. The domains were written with $11-13 \mathrm{~mW}, 1.85 \mathrm{MHz}, 100 \mathrm{nsec}$ laser pulse at a linear velocity of $7.54 \mathrm{~m} / \mathrm{sec}$. The 


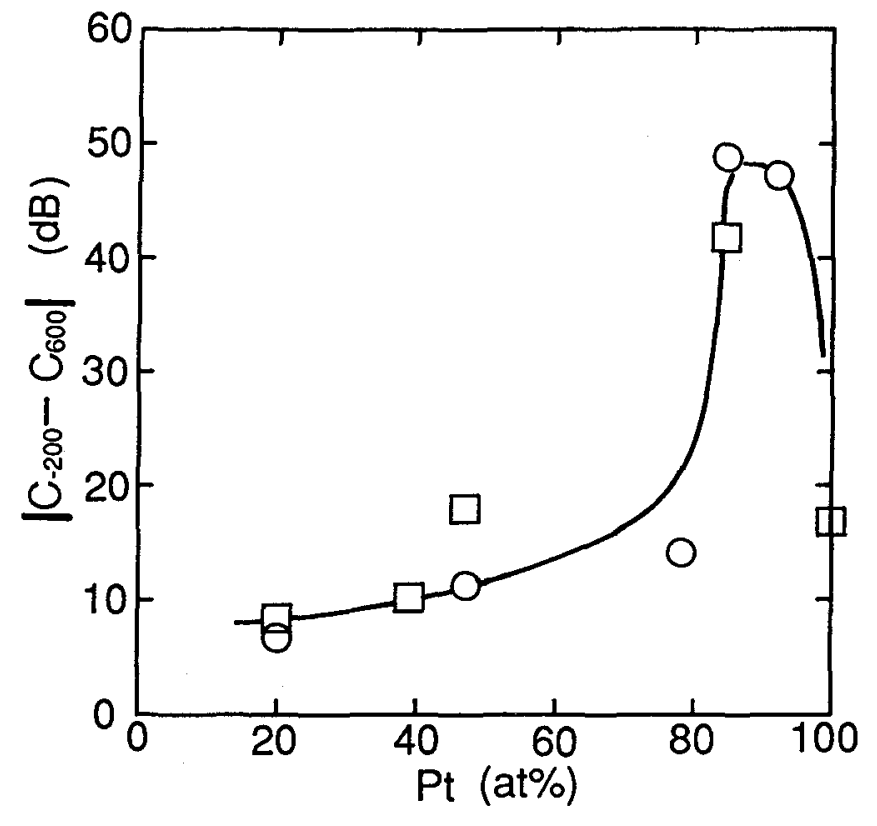

Fig.5 Relation between $\mid C_{-200}-C_{600}$ land Pt content of PtCo capping layer. $C_{x}$ means the carrier level at $x \mathrm{Oe}$. $O, \square$ are the data for PtCo layer thickness, $50 \mathrm{~nm}, 20 \mathrm{~nm}$, respectively.

dependence of the laser-written mark size upon the external field is quite weak, and the written marks consist of several microdomains near zero field[4]. In the disk $\mathrm{C}$, microdomains in a wide range of positive and negative fields are present around zero field. Whereas in the disk $A$, such multiple domains disappear in the relatively weak negative field. It should be noted that the PtCo capping layer suppresses the nucleation of microdomains in the interior of the written mark. Here, we prepared another disk with an insertion layer, and studied the effect of insertion of non-magnetic nitride between PtCo and TbFeCo. Figure 6 shows the field dependence of the carrier and noise levels for the nitride inserted disk compared with those for the disk A. It is seen that the insertion of nonmagnetic nitride layer with a thickness of only $4 \mathrm{~nm}$

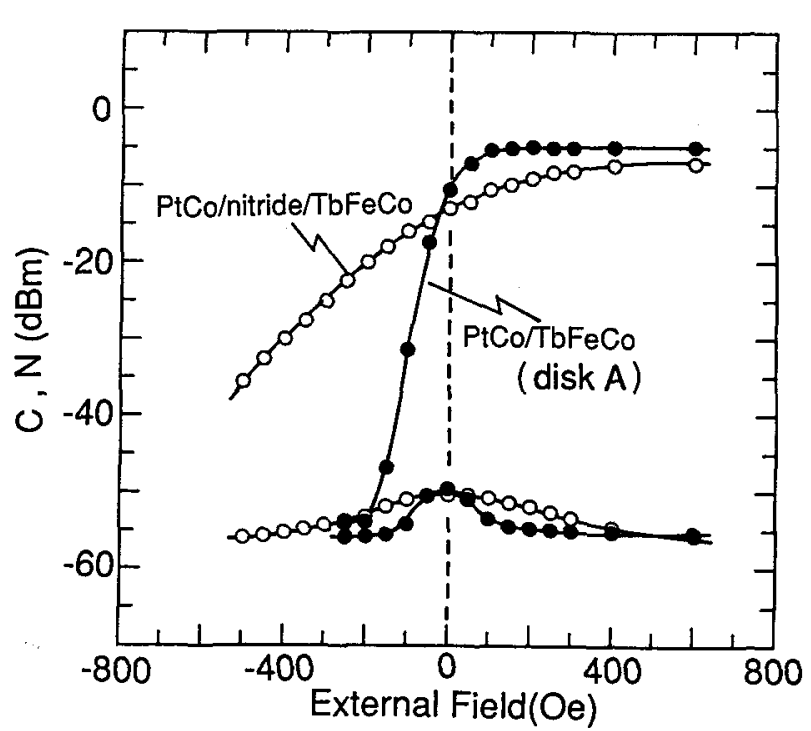

Fig.6 External field dependence of the carrier and noise levels for the disk using $\mathrm{PtCo} /$ nitride/TbFeCo triple layered film.

between PtCo layer and $\mathrm{TbFeCo}$ layer inhibits the capping layer effect. This result suggests that the direct contact between PtCo and TbFeCo plays a decisive role in the above mentioned phenomenon. Figure 7 shows perpendicular $\mathrm{M}-\mathrm{H}$ hysteresis loops of $\mathrm{Pt}_{84} \mathrm{Co}_{16} / \mathrm{TbFeCo}$ double layered film(c) and $\mathrm{Pt}_{84-}$ Co16/nitride(4nm)/TbFeCo triple layered film(d) together with those of a single $\mathrm{TbFeCo}$ film(a) and a single $\mathrm{Ptia}_{84} \mathrm{Co}_{16}$ film(b). The narrow arrow in the upper half of each box in the figure(c),(d) indicates the direction of PtCo layer's magnetization, and that in the lower half shows the magnetization direction of the transition metals $(\mathrm{Fe}, \mathrm{Co})$ in the $\mathrm{TbFeCo}$ layer. The open arrow in the lower half represents the direction of $\mathrm{Tb}$ magnetization of the TbFeCo layer.The M-H loop of the triple layered film is a simple superposition of those of - its $\mathrm{TbFeCo}$ and PtCo films, whereas for the double layered film without nitride insertion, the loop shows a gradual change at the shoulder part indi-

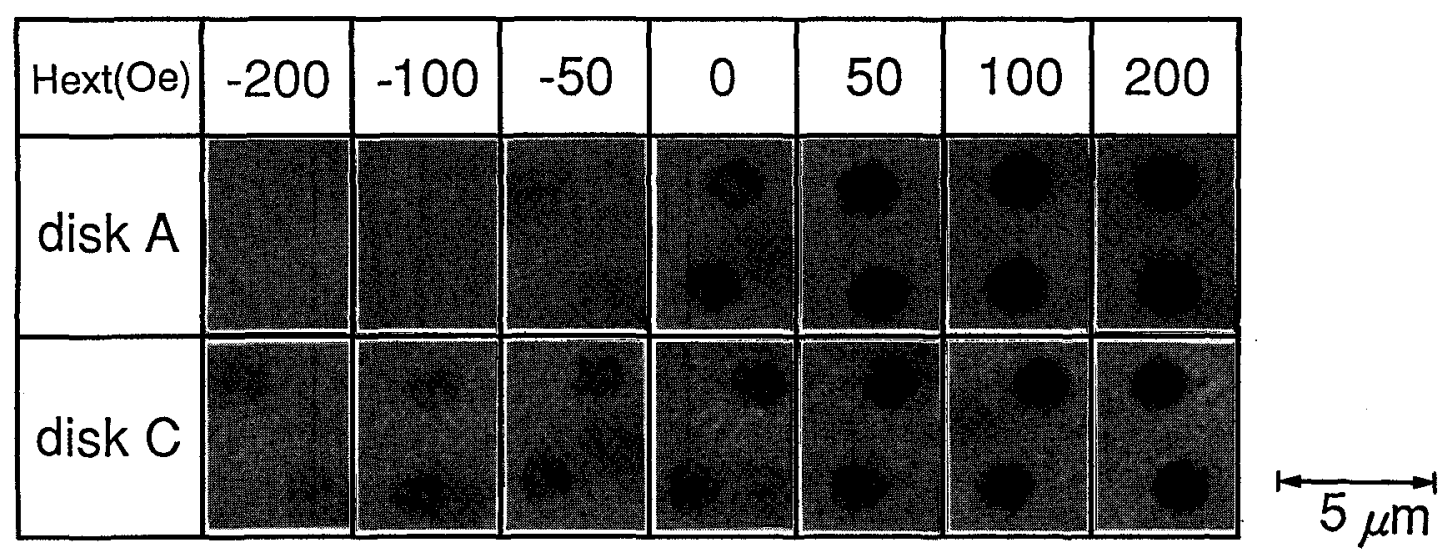

Photo.1 External field dependence of written domains for disk $A$ and disk $C$. 

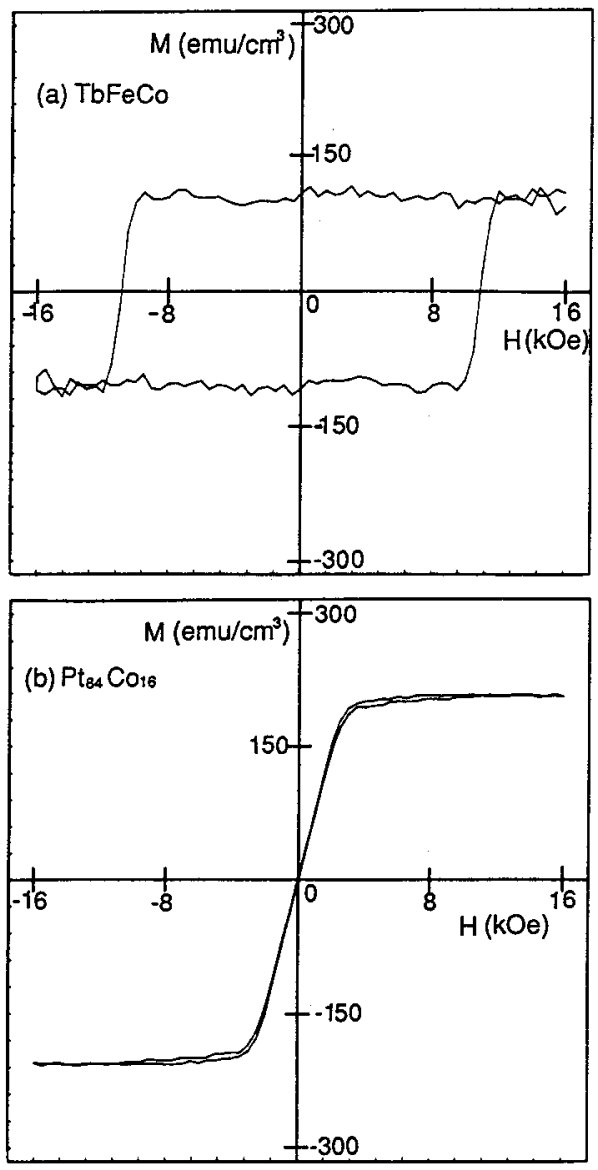
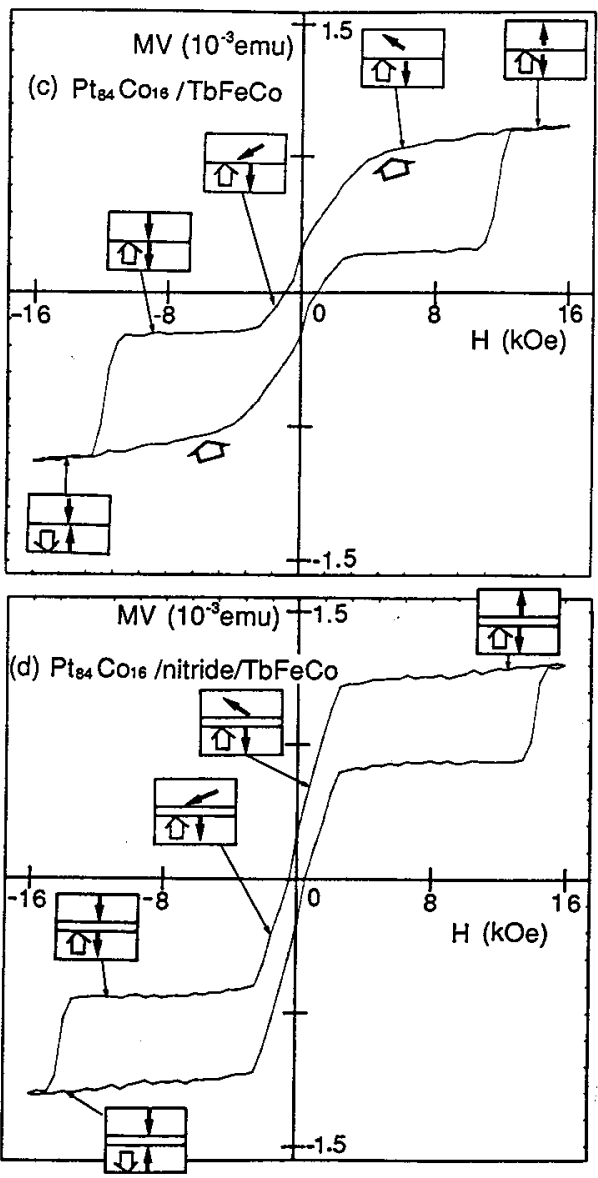

Fig.7 Perpendicular M-H hysteresis loops as follows : (a) TbFeCofilm,(b) $\mathrm{Pt}_{84} \mathrm{CO}_{16}$ film, (c) $\mathrm{Pt}_{84} \overline{\mathrm{Co}_{16}} / \mathrm{TbFeCo}$ double layered film,(d) $\mathrm{Pt}_{84} \mathrm{Co}_{16} /$ nitride/TbFeCo triple layered film.

cated by the bold arrows. This gradual change will be attributed to the decrease of the perpendicular component in PtCo magnetization with decreasing external field due to exchange coupling between $\mathrm{PtCo}$ and $\mathrm{TbFeCo}$. In other words, the insertion of non-magnetic layer between them interrupts the magnetic exchange coupling. Taking into consideration of the facts mentioned above, we propose that the capping layer may act to increase the effective domain wall energy and the nucleation field of the reverse domains through exchange coupling between the two layers.

\section{CONCLUSIONS}

A new Magneto-Optical disk using a TbFeCo film capped with in-plane magnetizing PtCo alloy film was studied. It was found that a capping layer of $\mathrm{PtCo}$ alloy film with $80-90$ at\% $\mathrm{Pt}$ substantially reduces the switching field in the MO disk. This PtCo capping layer effect is very useful for the overwriting schemes such as in field modulation type and two beam type. The detailed investigation of the marks written by a laser beam revealed that the reduction of the switching field is strongly related to the suppression of the microdomain nucleation during the switching process. This will be attributed to the presumable increase of the effective domain wall energy and the nucleation field of the reverse domains through exchange coupling between the $\mathrm{TbFeCo}$ recording layer and the PtCo capping layer.

\section{REFERENCES}

[1] J. A. Aboaf, S. R. Herd and E. Klokholm : IEEE Trans. Magn. MAG-19(4),1514(1983).

[2] M. Kitada and N. Shimizu:J. Appl. Phys.,54(12), 7089(1983).

[3] M. Hansen and K. Anderko: "Constitution of Binary Alloys. Second Edition", 1958, pp.492

[4] J. C. Suits, D. Ruger and C. J. Lin : J. Appl. Phys.,64(1),252(1988). 